\title{
Correlating mRUST scoring with fracture healing in the mouse model
}

Anthony C. McGuire ${ }^{1}$, Adam Knox ${ }^{1}$, Caio De Andrade Staut ${ }^{1}$, Melissa A. Kacena ${ }^{1,2}$, Roman M. Natoli ${ }^{1}$, and Christopher D. Collier ${ }^{1}$

1Department of Orthopaedic Surgery, Indiana University School of Medicine, IN, USA

${ }^{2}$ Richard L. Roudebush VA Medical Center, IN, USA

Background/Objective: Long bone fractures are an expensive and frequent cause of disability in humans. Research seeking to accelerate and improve the healing process is more essential than ever. Animal models, mice especially, provide an inexpensive and reproducible model of in vivo fracture healing. However, many measures of murine fracture healing outcomes are either expensive or destructive, limiting their ability to be translated to clinical studies. We seek to determine how these measures such as biomechanics, $\mu \mathrm{CT}$, and histology correlate to the relatively new, inexpensive, and non-destructive method of mRUST scoring in a mouse model.

Methods: One hundred and thirty-five, 12-week old male C57BL6/J mice were divided into nine groups of 15 mice. Mice underwent a surgically created, femoral fracture. At biweekly timepoints, anteroposterior and lateral radiographs were taken, and 15 mice were sacrificed at each time point $(7,10,14,17,21,24,28,35$, and 42 days post-surgery) for biomechanical, $\mu \mathrm{CT}$, and histological analyses. The modified Radiographic Union Scale for Tibial fractures (mRUST scoring) provides a score based on the visualization of a callus and fracture line in four cortices on the radiographs. Data analysis will be performed to determine the degree of correlation between mRUST scoring and other fracture healing outcomes.

Results/Conclusion: Data collection in this experiment is still forthcoming. Upon successful completion of this project, we will have established numerical correlations between mRUST scoring and other fracture healing outcomes, such as biomechanics, $\mu \mathrm{CT}$ microarchitecture, and histology. These correlations will provide a powerful tool in future mouse fracture healing studies, as data on the state and strength of fracture repair could be determined by simple radiograph.

Scientific/Clinical Policy Impact and Implications: This study will both provide future murine fracture studies with an inexpensive and non-destructive method of assessment that is more directly translatable to human fracture studies. 